\title{
Rate and Prognosis of Patients under Conscious Sedation Requiring Emergent Intubation during Neuroendovascular Procedures
}

\author{
A.E. Hassan, U. Akbar, S.A. Chaudhry, W.G. Tekle, R.P. Tummala, G.J. Rodriguez, and A.I. Qureshi
}

\begin{abstract}
BACKGROUND AND PURPOSE: Neuroendovascular procedures are performed with the patient under conscious sedation (local anesthesia) in varying numbers of patients in different institutions, though the risk of unplanned conversion to general anesthesia is poorly characterized. Our aim was to ascertain the rate of failure of conscious sedation in patients undergoing neuroendovascular procedures and compare the in-hospital outcomes of patients who were converted from conscious sedation to general anesthesia with those whose procedures were initiated with general anesthesia.
\end{abstract}

MATERIALS AND METHODS: All patients who had an endovascular procedure initiated under general anesthesia or conscious sedation were identified through a prospective data base maintained at 2 comprehensive stroke centers. Patient clinical and procedural characteristics, in-hospital deaths, and favorable outcomes (modified Rankin Scale score, 0-2) at discharge were ascertained.

RESULTS: Nine hundred seven endovascular procedures were identified, of which 387 were performed with the patient under general anesthesia, while 520 procedures were initiated with conscious sedation. Among procedures initiated with intent to be performed under conscious sedation, 9 (1.7\%) procedures required emergent conversion to general anesthesia. Favorable clinical outcome and in-hospital mortality in patients requiring emergent conversion from conscious sedation to general anesthesia and in those with procedures initiated with general anesthesia were not statistically different ( $42 \%$ versus $50 \%, P=.73$ and $17 \%$ versus $13 \%, P=1.00$, respectively).

CONCLUSIONS: In our study, there was a very low rate of conscious sedation failure and associated adverse outcomes among patients undergoing neuroendovascular procedures. Proper patient selection is important if procedures are to be performed with the patient under conscious sedation. Limitations of the methodology used in our study preclude us from offering specific recommendations regarding when to use a specific anesthetic protocol.

ABBREVIATIONS: mRS = modified Rankin Scale; ICU = intensive care unit

ontroversy exists regarding the type of anesthesia required in patients undergoing neuroendovascular procedures. Some operators advocate the use of local anesthesia and intravenous sedation known as conscious sedation, while others argue that these interventions are best performed with the patient under general anesthesia. One study showed that initiation of an inter-

Received May 8, 2012; accepted after revision August 30.

From the Zeenat Qureshi Stroke Research Center (A.E.H., S.A.C., W.G.T., G.J.R., A.I.Q.), Departments of Neurology (A.E.H., S.A.C., W.G.T., R.P.T., G.J.R., A.I.Q.) and Neurosurgery (R.P.T., A.I.Q.), University of Minnesota, Minneapolis, Minnesota; Hennepin County Medical Center (A.E.H., W.G.T., G.J.R., A.I.Q.), Minneapolis, Minnesota; Department of Neurology (U.A.), University of Medicine and Dentistry, New Jersey/Cooper Medical Center, Camden, New Jersey; Neurology, Radiology, and Neurosurgery (A.E.H.), University of Texas Health Science Center, San Antonio, Texas; and Endovascular Surgical Neuroradiology, Neurocritical Care, and Clinical Neuroscience Research (A.E.H.), Valley Baptist Medical Center, Harlingen, Texas.

Please address correspondence to Ameer E. Hassan, DO, Department of Neurology, Zeenat Qureshi Stroke Research Center, University of Minnesota, 12-100 PWB 516 Delaware St SE, Minneapolis, MN 55455; e-mail: ameerehassan@gmail.com

http://dx.doi.org/10.3174/ajnr.A3385 vention for anterior circulation stroke with the patient under general anesthesia varied widely among centers, ranging from $0 \%$ to $100 \%$, with the average being $44 \% .{ }^{1}$ A recent survey reported that a large majority of neurointerventionalists prefer general anesthesia as the intraprocedural technique of choice. ${ }^{2}$

Although such decisions depend on personal preference, experience, and institutional protocols, some studies suggest that certain modalities of sedation may prolong the hospital stay and worsen outcome. Concern for increased risk of aspiration and potential airway injury with emergent intubation ${ }^{3}$ in a procedure initiated with conscious sedation, especially if thrombolytic therapy or anticoagulation has been used, may bias operators toward general anesthesia at the onset of the procedure. However, there is insufficient evidence to suggest that such conversion is a common occurrence or that it is detrimental to the patient. We sought to determine the frequency and prognosis of patients converted from conscious sedation to general anesthesia during neuro- 
Table 1: Endovascular procedures according to type of anesthesia used

\begin{tabular}{lccc}
\multicolumn{1}{c}{ Variables } & $\begin{array}{c}\text { Procedures Started } \\
\text { under General } \\
\text { Anesthesia }\end{array}$ & $\begin{array}{c}\text { Procedures Started } \\
\text { under Conscious } \\
\text { Sedation }\end{array}$ & $\begin{array}{c}\text { Failure of Conscious } \\
\text { Sedation Conversion } \\
\text { Rate }\end{array}$ \\
\hline $\begin{array}{l}\text { Total procedures } \\
\text { Type of procedure }\end{array}$ & 387 & 526 & $9 / 526(1.7 \%)$ \\
Endovascular treatment of cerebral vasospasm & $88(23 \%)$ & $81(15.4 \%)$ & $2(2.5 \%)$ \\
Extracranial carotid artery stent placement & $11(3 \%)$ & $172(32.7 \%)$ & $1(0.6 \%)$ \\
Intracranial angioplasty and/or stent placement & $30(8 \%)$ & $51(9.7 \%)$ & $1(2.0 \%)$ \\
Embolization of ruptured aneurysm & $86(22 \%)$ & $21(3.9 \%)$ & $1(4.8 \%)$ \\
Embolization of unruptured aneurysm & $53(14 \%)$ & $28(5.3 \%)$ & $1(3.6 \%)$ \\
Endovascular treatment of acute ischemic stroke & $50(13 \%)$ & $86(16.3 \%)$ & $3(3.5 \%)$ \\
Embolization of AVM/epistaxis & $69(18 \%)$ & $87(16.5 \%)$ & $0(0 \%)$ \\
No. of procedures per patient & & & \\
1 & $199(75 \%)$ & & \\
2 & $38(14 \%)$ & & \\
$\geq 3$ & $28(11 \%)$ & & \\
\hline
\end{tabular}

interventional procedures at 2 academic comprehensive stroke centers.

\section{MATERIALS AND METHODS}

This retrospective study identified all patients undergoing neuroendovascular procedures at 2 academic centers following review of a procedure log from 2006 to 2010. All patients who had an endovascular procedure that was initiated under general anesthesia or conscious sedation, with the exception of diagnostic angiography, were included in the current study. Demographics including age, sex, and race/ethnicity were collected from admission registration profiles. Clinical data were obtained from hospital records, including the number and type of neuroendovascular procedures performed and the procedural outcomes, which were determined by modified Rankin Scale score and death at hospital discharge. We collected information regarding the cardiovascular risk factors (mentioned in the medical records; eg, hypertension, dyslipidemia, diabetes mellitus, cigarette smoking, atrial fibrillation, and coronary artery disease). The information was presumed to be absent if we were unable to find clear documentation. We were unable to characterize the adequacy of risk-factor control such as cessation of cigarette smoking and control of hypertension and hyperlipidemia because of a lack of standardized documentation.

\section{Statistical Analysis}

Statistical analysis was performed by using SAS 9.1 software (SAS Institute, Cary, North Carolina). Descriptive statistics were expressed as means with SDs and frequency (percentages). Continuous and categoric variables were compared by using ANOVA and $\chi^{2}$ tests, respectively. We assessed whether cardiovascular risk factors were similar between patients in whom procedures were started under general anesthesia and those who required emergent conversion from conscious sedation to general anesthesia. We also compared the rates of favorable clinical outcome and in-hospital mortality between the 2 study groups.

\section{RESULTS}

Nine hundred seven endovascular procedures were identified during the study period, of which 387 were performed with the patient under general anesthesia, while 520 procedures were initiated with the intent to perform them under conscious sedation.
Among procedures with the intent to perform under conscious sedation, $9(1.7 \%)$ were emergently converted to general anesthesia. Table 1 summarizes the number and types of procedures performed and the respective conversion rates. Endovascular treatment of cerebral vasospasm ( 88 cases, $23 \%$ of total cases) and embolization of ruptured aneurysms (86 cases, 22\%) were the most common interventions performed with the patient under general anesthesia.

Two hundred sixty patients underwent 387 procedures that were initiated with general anesthesia, while 8 patients underwent 9 procedures and were emergently converted from conscious sedation to general anesthesia. The median age was $53 \pm 16$ years, and 1 patient (13\%) was a woman. Cardiovascular risk factors between the 2 groups were similar (Table 2). Detailed information regarding the type of procedure, cardiovascular risk factors, stroke severity at admission, and discharge outcome is presented in Table 3. Overall, the most common reason for conversion was the inability of the patient to follow commands and/or maintain immobility during the procedure ( 7 of 9 conversions). One patient vomited during endovascular treatment of acute ischemic stroke and was intubated in response to aspiration, and another patient had intraprocedural rupture while undergoing embolization of an intracranial aneurysm and was intubated due to a deteriorating level of consciousness. Favorable clinical outcome and in-hospital mortality in general anesthesia-only patients and those with emergent conversion from conscious sedation to general anesthesia were not statistically different ( $42 \%$ versus $50 \%$, $P=.73 \%$ and $17 \%$ versus $13 \%, P=1.00$, respectively).

We performed an exploratory analysis comparing the admission NIHSS scores in patients undergoing endovascular treatment under conscious sedation with those of patients treated with general anesthesia from initiation. The mean admission NIHSS score $(12.7 \pm 5.3)$ in patients undergoing endovascular treatment successfully under conscious sedation was similar to that in patients who failed conscious sedation $(10.0 \pm 4.4)$ and lower than that in patients who were treated under general anesthesia from initiation $(18.5 \pm 7.7)$.

\section{DISCUSSION}

Due to the lack of standardization in large device trials, ${ }^{4,5}$ many neurointerventionalists are reluctant to treat patients under conscious sedation, especially due to the potential risk of airway dam- 
Table 2: Comparison of baseline characteristics and outcomes among patients with conscious sedation and planned and unplanned general anesthesia for endovascular procedures

\begin{tabular}{|c|c|c|c|c|}
\hline & $\begin{array}{l}\text { Patients who Underwent } \\
\text { Endovascular Procedures } \\
\text { under Conscious Sedation }\end{array}$ & $\begin{array}{l}\text { Patients who Underwent } \\
\text { Endovascular Procedures under } \\
\text { Planned General Anesthesia }\end{array}$ & $\begin{array}{l}\text { Patients Who Underwent } \\
\text { Endovascular Procedures under } \\
\text { Unplanned General Anesthesia }\end{array}$ & $\begin{array}{c}P \\
\text { Value }^{\mathrm{a}, \mathrm{b}}\end{array}$ \\
\hline No. of patients & 387 & 260 & 8 & \\
\hline Mean age (yr) & $60.2 \pm 16.7$ & $56 \pm 18$ & $53 \pm 16$ & .09 \\
\hline Women & $192(49.6 \%)$ & $134(51 \%)$ & $1(13 \%)$ & .0260 \\
\hline \multicolumn{5}{|l|}{ Risk factors } \\
\hline Hypertension & $235(60.7 \%)$ & $142(55 \%)$ & $6(75 \%)$ & .0979 \\
\hline Hyperlipidemia & $166(42.8 \%)$ & $60(23 \%)$ & $3(38 \%)$ & $<.0001$ \\
\hline Diabetes mellitus & $91(23.5 \%)$ & $39(15 \%)$ & $1(13 \%)$ & .0303 \\
\hline Coronary artery disease & $65(16.8 \%)$ & $39(15 \%)$ & $1(13 \%)$ & .8057 \\
\hline Congestive heart failure & $32(8.3 \%)$ & $23(9 \%)$ & $1(13 \%)$ & .8394 \\
\hline Atrial fibrillation & $40(10.3 \%)$ & $26(10 \%)$ & $0(0 \%)$ & .6630 \\
\hline Previous stroke & $67(17.3 \%)$ & $30(12 \%)$ & $1(13 \%)$ & .0876 \\
\hline \multicolumn{5}{|l|}{ Clinical outcome } \\
\hline Favorable outcome (mRS, 0-2) & $264(68.2 \%)$ & 109 (42\%) & $4(50 \%)$ & $<.0001$ \\
\hline Death & $17(4.4 \%)$ & $45(17 \%)$ & $1(13 \%)$ & $<.0001$ \\
\hline
\end{tabular}

a $P<.025$, adjusting for multiple comparisons using Bonferroni correction.

${ }^{b}$ Note that the possibility of type II error exists in calculating significance due to the small number of patients.

age and aspiration from an emergent intubation. ${ }^{3,6}$ This reason was rated by $43 \%$ of interventionalists as the most concerning complication in a survey involving 49 physicians nationwide. ${ }^{2}$ Our study demonstrates that the rate of emergent conversion from conscious sedation to general anesthesia was very low $(1.7 \%)$. Emergent conversion to general anesthesia was not associated with worse outcomes than those observed in planned general anesthesia.

Previous smaller studies assessing the rates of conversion to general anesthesia during acute ischemic stroke treatment, aneurysm embolization, and carotid artery stent placement have reported rates varying from $1.3 \%$ to $3 \% .^{7-9}$ According to a study by Jumaa et al, ${ }^{8} 2$ patients (2.7\%) were emergently intubated because of conscious sedation failure among 73 patients with acute isch- emic stroke treated endovascularly with conscious sedation. In elective carotid stent placements, Chamczuk et $\mathrm{al}^{9}$ found that only 2 of $63(3 \%)$ patients had to be converted to general anesthesia. Qureshi et $\mathrm{al}^{7}$ demonstrated successful embolization of ruptured and unruptured aneurysms with patients under conscious sedation with low complication rates. Two conversions occurred in 150 aneurysm cases that underwent embolization with Guglielmi detachable coils (Boston Scientific, Natick, Massachusetts). Both ICU and hospital mean length of stay were significantly higher in the general anesthesia cohort compared with conscious sedation in both ruptured ( 11 versus 17 and 20 versus 30 days) and unruptured ( 3 versus 11 and 6 versus 20 days) groups. Morbidity and mortality rates for ruptured $(1.6 \%$ and $3 \%)$ and unruptured $(2 \%$ and $0 \%$ ) aneurysms were lower than or similar to those in previ-

Table 3: Demographic, clinical, and procedural characteristics of patients with conscious sedation failure requiring emergent intubation during neuroendovascular procedures

\begin{tabular}{|c|c|c|c|c|c|c|c|c|}
\hline No. & $\begin{array}{l}\text { Age } \\
(y r) / \\
\text { Sex }\end{array}$ & $\begin{array}{l}\text { Type of } \\
\text { Procedure }\end{array}$ & Medical History & $\begin{array}{c}\text { ASA } \\
\text { Grade }^{a, b}\end{array}$ & $\begin{array}{l}\text { GCS } \\
\text { Score }^{b}\end{array}$ & $\begin{array}{l}\text { Reason for } \\
\text { Conversion }\end{array}$ & $\begin{array}{c}\text { Total } \\
\text { Procedural } \\
\text { Time (min) }\end{array}$ & $\begin{array}{c}\text { mRS at } \\
\text { Discharge }\end{array}$ \\
\hline 1 & $47 / M$ & $\begin{array}{l}\text { Endovascular treatment of } \\
\text { cerebral vasospasm }\end{array}$ & Hypertension & 4 & 13 & Movement & 79 & 1 \\
\hline 2 & $56 / M$ & $\begin{array}{l}\text { Extracranial carotid artery } \\
\text { stent placement }\end{array}$ & $\begin{array}{l}\text { Hypertension, hyperlipidemia, } \\
\text { previous stroke }\end{array}$ & 2 & 14 & Movement & 68 & 2 \\
\hline 3 & $45 / M$ & $\begin{array}{l}\text { Endovascular treatment of } \\
\text { acute ischemic stroke }\end{array}$ & Hypertension, hyperlipidemia & 3 & 13 & $\begin{array}{l}\text { Vomiting } \\
\text { (airway protection) }\end{array}$ & 172 & 2 \\
\hline 4 & $33 / M$ & $\begin{array}{l}\text { Embolization of ruptured } \\
\text { aneurysm }\end{array}$ & Hypertension & 4 & 13 & Re-ruptured aneurysm & 198 & 4 \\
\hline 5 & $86 / M$ & $\begin{array}{l}\text { Intracranial angioplasty } \\
\text { and/or stent placement }\end{array}$ & $\begin{array}{l}\text { Hypertension, diabetes } \\
\text { mellitus, congestive heart } \\
\text { failure }\end{array}$ & 2 & 15 & Movement & 424 & 6 \\
\hline 6 & $61 / M$ & $\begin{array}{c}\text { Endovascular treatment of } \\
\text { acute ischemic stroke }\end{array}$ & $\begin{array}{l}\text { Hypertension, hyperlipidemia, } \\
\text { coronary artery disease }\end{array}$ & 3 & 13 & Movement & 115 & 5 \\
\hline 7 & $50 / F$ & $\begin{array}{l}\text { Embolization of unruptured } \\
\text { aneurysm }\end{array}$ & None & 1 & 15 & Movement & 372 & 2 \\
\hline 8 & $50 / F$ & $\begin{array}{l}\text { Endovascular treatment of } \\
\text { acute ischemic stroke }\end{array}$ & None & 1 & 15 & Movement & 62 & 2 \\
\hline 9 & $46 / M$ & $\begin{array}{l}\text { Endovascular treatment of } \\
\text { cerebral vasospasm }\end{array}$ & None & 4 & 14 & Movement & 345 & 4 \\
\hline
\end{tabular}

Note:-ASA indicates American Society of Anesthesiologists; GCS, Glasgow Coma Scale; mRs, modified Rankin scale.

${ }^{a}$ American Society of Anesthesiologists grade: $1=$ a healthy patient; 2 = patient with mild systemic disease; $3=$ patient with severe systemic disease; $4=$ patient with severe systemic disease that is a constant threat to life; $5=$ moribund patient who is not expected to survive without the operation; $6=$ declared brain-dead patient whose organs are being removed for donor purposes.

${ }^{\mathrm{b}}$ Prior to procedure. 
ous studies in which general anesthesia was used $(3 \%-17 \%$ and $0 \%-8 \%$, respectively), though direct comparison is precluded by differences in patient characteristics.

Although some case series have reported the safety of specific individual procedures with the patient under conscious sedation, ${ }^{1,7-12}$ large case series have only reported upon the endovascular treatment of acute ischemic stroke. ${ }^{1,8}$ General anesthesia was associated with a longer ICU stay, higher rates of pneumonia, larger infarcts, and worse clinical outcome in studies conducted in patients undergoing acute ischemic stroke treatment. ${ }^{8}$ Avoidance of general anesthesia was also associated with reduced odds of cardiovascular complications 30 days after carotid endarterectomy. ${ }^{13,14}$ Presumably, the higher rate is related to occurrence of anesthetic-related deaths and severe cardiovascular adverse events. ${ }^{15,16}$ Common adverse events associated with general anesthesia reported by Forrest et $\mathrm{al}^{15,16}$ included tachycardia (41\%), hypotension (31\%), hypertension (27\%), bradycardia (19\%), and ventricular arrhythmias (6\%). Detection of clinical events related to iatrogenic thromboembolism is prevented by prolonged recovery from anesthesia; therefore, the possibility of early revascularization is lost. ${ }^{17}$ Abou-Chebl et $\mathrm{al}^{10}$ reported that in 37 patients who underwent intracranial angioplasty and stent placement under conscious sedation, operators modified the interventional technique in $61 \%$ of patients on the basis of real-time patient reporting of intraprocedural symptoms.

Previous studies have demonstrated significant hospital cost reduction by using conscious sedation instead of general anesthesia. In addition to the procedural costs of anesthetic agents, anesthesia personnel, and mechanical ventilation, considerable expenses can be avoided with reduction of hospital and ICU stays. At some institutions, general anesthesia for a 3-hour embolization procedure may cost $\$ 1350^{7}$; the avoidance this fee reduced mean total hospital cost at 1 institution by $41 \% .{ }^{18}$

Even so, a technically successful procedure (ie, revascularization after ischemic stroke or technically successful aneurysm embolization) does not necessarily correlate with good outcome. Advocates for conscious sedation argue that the advantage of realtime input from the patient and the clinical examination outweigh the risk of complications from patient mobility. ${ }^{19,20}$ Other potential disadvantages of performing these procedures with the patient under general anesthesia include induction-related hypotension, delay in procedure initiation, the need for additional personnel and equipment in the angiography suite, and inherent delay in obtaining a postoperative neurologic examination.

Patient selection is important for successful completion of neuroendovascular procedures with the patient under conscious sedation. Procedural type, anticipated duration, and patient characteristics are important in identifying appropriate patients for conscious sedation. Certain procedures such as extracranial carotid artery stent placement infrequently require general anesthesia because of the limited impact of patient motion, head immobility, and hemodynamic control on procedural success. ${ }^{21}$ However, a complex aneurysm that requires real-time assessment in multiple angiographic projections or a very small aneurysm is best treated with the patient under general anesthesia because even small-magnitude patient movement can interfere with the success of the procedure. Similarly, anticipated prolonged dura- tion in a case of complex dural arteriovenous fistula (several hours of embolization time) may be better performed with the patient under general anesthesia. Patient-related factors such as a poor level of consciousness, communicative disorders, or difficulty in remaining immobile due to a chronic pain disorder need to be evaluated during preprocedural clinical assessment or during a preceding diagnostic cerebral angiogram with the patient under conscious sedation.

Some limitations should be considered before interpretation of our results. Due to a small number of patients in each intervention group, the precision of the estimation regarding conversion rates about individual subgroups defined by procedure type is not optimal. There was also no long-term follow-up in many patients, thereby limiting our ability to determine the long-term consequences of the choice of anesthetic technique. Conversely, the retrospective nature of the study prevented bias in choosing a sedation technique because the practitioners were unaware that this issue would be studied. Thus, decisions were made in realtime on the basis of the clinical data available to the neurointerventionalists. A randomized controlled trial with a standardized inclusion/exclusion protocol is needed to determine the comparative benefits of procedures performed under general anesthesia or conscious sedation.

\section{CONCLUSIONS}

In our study, there was a very low rate of conscious sedation failure and associated adverse outcomes among patients undergoing neuroendovascular procedures. Proper patient selection is important if procedures are to be performed under conscious sedation. Limitations of the methodology used in our study preclude us from offering specific recommendations regarding when to use a specific anesthetic protocol.

\section{ACKNOWLEDGMENT}

The authors would like to thank Sarwat Gilani for her assistance in this study.

Disclosures: Ramachandra P. Tummala—UNRELATED: Consultancy: Lake Regional Medical, Comments: consultant to review new devices, Payment for Lectures (including service on Speakers Bureaus): lecturer at Midwest Neuroendovascular Fellow Course, University of lowa, May 2011; Dr. A.I. Qureshi: funding from the National Institutes of Health RO1-NS44976-01A2 (medication provided by ESP Pharma), American Heart Association Established Investigator Award 0840053N, National Institutes of Health U01-NS062091-01A2, and the Minnesota Medical Foundation, Minneapolis, Minnesota.

\section{REFERENCES}

1. Abou-Chebl A, Lin R, Hussain MS, et al. Conscious sedation versus general anesthesia during endovascular therapy for acute anterior circulation stroke: preliminary results from a retrospective, multicenter study. Stroke 2010;41:1175-79

2. McDonagh DL, Olson DM, Kalia JS, et al. Anesthesia and sedation practices among neurointerventionalists during acute ischemic stroke endovascular therapy. Front Neurol 2010;1:118

3. Li J, Murphy-Lavoie H, Bugas C, et al. Complications of emergency intubation with and without paralysis. Am J Emerg Med 1999;17:141-43

4. Penumbra Pivotal Stroke Trial Investigators. The Penumbra Pivotal Stroke Trial: safety and effectiveness of a new generation of mechanical devices for clot removal in intracranial large vessel occlusive disease. Stroke 2009;40:2761-68 
5. Smith WS, Sung G, Saver J, et al. Mechanical thrombectomy for acute ischemic stroke: final results of the Multi Merci Trial. Stroke 2008;39:1205-12

6. Rosenberg M, Weaver J. General anesthesia. Anesth Prog 1991;38: 172-86

7. Qureshi AI, Suri MF, Khan J, et al. Endovascular treatment of intracranial aneurysms by using Guglielmi detachable coils in awake patients: safety and feasibility. J Neurosurg 2001;94:880-85

8. Jumaa MA, Zhang F, Ruiz-Ares G, et al. Comparison of safety and clinical and radiographic outcomes in endovascular acute stroke therapy for proximal middle cerebral artery occlusion with intubation and general anesthesia versus the nonintubated state. Stroke 2010;41:1180-84

9. Chamczuk AJ, Ogilvy CS, Snyder KV, et al. Elective stenting for intracranial stenosis under conscious sedation. Neurosurgery 2010;67: 1189-93, discussion 1194

10. Abou-Chebl A, Krieger DW, Bajzer CT, et al. Intracranial angioplasty and stenting in the awake patient. $J$ Neuroimaging 2006;16:216-23

11. Mori T, Kazita K, Mori K. Cerebral angioplasty and stenting for intracranial vertebral atherosclerotic stenosis. AJNR Am J Neuroradiol 1999;20:787-89

12. Ramee SR, Dawson R, McKinley KL, et al. Provisional stenting for symptomatic intracranial stenosis using a multidisciplinary approach: acute results, unexpected benefit, and one-year outcome. Catheter Cardiovasc Interv 2001;52:457-67
13. Allen BT, Anderson CB, Rubin BG, et al. The influence of anesthetic technique on perioperative complications after carotid endarterectomy. J Vasc Surg 1994;19:834-42, discussion 842-43

14. Tangkanakul C, Counsell CE, Warlow CP. Local versus general anaesthesia in carotid endarterectomy: asystematic review of the evidence. Eur J Vasc Endovasc Surg 1997;13:491-99

15. Forrest JB, Cahalan MK, Rehder K, et al. Multicenter study of general anesthesia. II. Results. Anesthesiology 1990;72:262-68

16. Forrest JB, Rehder K, Cahalan MK, et al. Multicenter study of general anesthesia. III. Predictors of severe perioperative adverse outcomes. Anesthesiology 1992;76:3-15

17. Mingus ML. Recovery advantages of regional anesthesia compared with general anesthesia: adult patients. J Clin Anesth 1995;7:628-33

18. Back MR, Harward TR, Huber TS, et al. Improving the cost-effectiveness of carotid endarterectomy. J Vasc Surg 1997;26:456-62, discussion 463-64

19. Gupta R. Local is better than general anesthesia during endovascular acute stroke interventions. Stroke 2010;41:2718-19

20. Molina CA, Selim MH. General or local anesthesia during endovascular procedures: sailing quiet in the darkness or fast under a daylight storm. Stroke 2010;41:2720-21

21. Brekenfeld C, Mattle HP, Schroth G. General is better than local anesthesia during endovascular procedures. Stroke 2010;41: $2716-17$ 